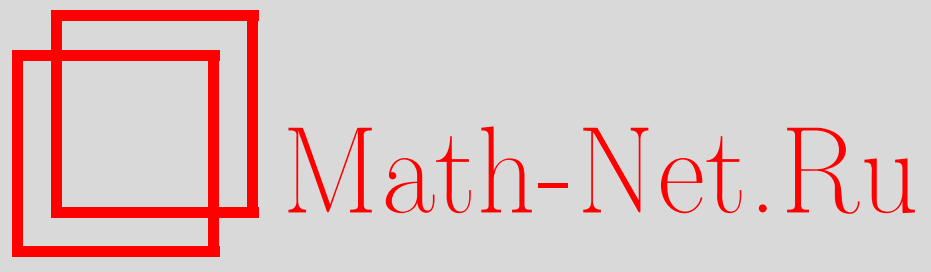

Д. Р. Гайфулин, Экстремальные значения континуантов, Матем. заметки, 2016, том 100, выпуск 2, 308-311

DOI: https://doi.org/10.4213/mzm11204

Использование Общероссийского математического портала Math-Net.Ru подразумевает, что вы прочитали и согласны с пользовательским соглашением http://www . mathnet.ru/rus/agreement

Параметры загрузки:

IP: 54.172 .240 .79

26 апреля 2023 г., 14:48:13

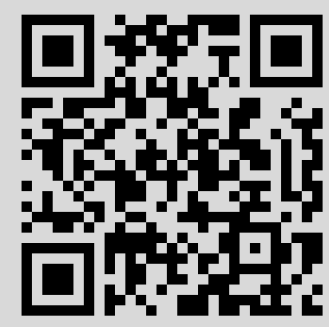




\section{Экстремальные значения континуантов}

\section{Д. Р. Гайфулин}

Ключевые слова: континуанты, цепные дроби.

DOI: $10.4213 / \mathrm{mzm} 11204$

1. Введение. Задачи на поиски экстремумов континуантов по различным множествам встречаются в ряде теоретико-числовых задачах. Примером может служить исследование производной функции Минковского ? $(x)$ и ее обобщений, начатое в [1] и развитое в работах [2], [3]. Большой класс задач, посвященный поиску экстремумов континуантов, рассмотрен в [4]. В данной статье дается окончательный ответ на одну из задач, поставленных в [4], а также решается ряд новых задач о максимумах и минимумах континуантов.

Для конечной последовательности натуральных чисел (возможно, нулевой длины) ( $a_{1}$, $\left.a_{2}, \ldots, a_{t}\right)$ континуант $\left\langle a_{1}, a_{2}, \ldots, a_{t}\right\rangle$ индуктивно определяется следующими соотношениями: $\langle\cdot\rangle=0,\left\langle a_{1}\right\rangle=a_{1}$,

$$
\left\langle a_{1}, a_{2}, \ldots, a_{t+1}\right\rangle=a_{t+1}\left\langle a_{2}, \ldots, a_{t}\right\rangle+\left\langle a_{1}, a_{2}, \ldots, a_{t-1}\right\rangle .
$$

Для конечной цепной дроби

$$
\left[a_{0} ; a_{1}, a_{2}, \ldots, a_{t}\right]=a_{0}+\frac{1}{a_{1}+\cdots+\frac{1}{a_{t}}}
$$

выполнено соотношение (см., например, [5])

$$
\left[a_{0} ; a_{1}, a_{2}, \ldots, a_{t}\right]=\frac{\left\langle a_{0}, a_{1}, a_{2}, \ldots, a_{t}\right\rangle}{\left\langle a_{1}, a_{2}, \ldots, a_{t}\right\rangle} .
$$

2. Обозначения и параметры. Все последовательности, рассматриваемые в данной статье, если не указано иное, по умолчанию состоят из натуральных чисел. Элементы континуантов мы часто будем называть неполными частными. Как правило, неполные частные будут обозначаться маленькими буквами $a, b, c, d, \ldots$. Большими буквами $A, B, C, D, \ldots$ мы будем обозначать конечные последовательности натуральных чисел. Для произвольных конечных последовательностей $A_{1}, A_{2}, \ldots, A_{n}$ через $\left(A_{1}, A_{2}, \ldots, A_{n}\right)$ обозначим последовательность, которую образуют все записанные друг за другом элементы $A_{1}$, $A_{2}, \ldots, A_{n}$. Обозначим последовательность $(\underbrace{a, a, \ldots, a}_{n \text { чисел }})$ через $a^{(n)}$. При нулевом $n$ положим эту последовательность пустой. Аналогично определим

$$
A^{(n)}=(\underbrace{A, A, \ldots, A}_{n \text { раз подряд }}) .
$$

Для непустой последовательности натуральных чисел $A=\left(a_{1}, a_{2}, \ldots, a_{t}\right)$ обозначим

$$
A_{-}=\left(a_{2}, \ldots, a_{t}\right), \quad A^{-}=\left(a_{1}, a_{2}, \ldots, a_{t-1}\right), \quad A_{-}^{-}=\left(a_{2}, a_{3}, \ldots, a_{t-1}\right) .
$$

Через $\vec{A}$ обозначим последовательность элементов $A$ в исходном порядке, а через $\overleftarrow{A}-$ в обратном. Нетрудно показать, что $\langle\overleftarrow{A}\rangle=\langle\vec{A}\rangle$

Работа выполнена при поддержке гранта РНФ (грант № 14-11-00433).

DOI: $10.4213 / \mathrm{mzm} 11204$

(C) Д. Р. ГАЙФулин, 2016 
Общеизвестно, что

$$
\langle A, B\rangle=\langle A\rangle\langle B\rangle+\left\langle A^{-}\right\rangle\left\langle B_{-}\right\rangle=\langle A\rangle\langle B\rangle(1+[0 ; \overleftarrow{A}][0 ; \vec{B}])
$$

Для $m \in \mathbb{N}_{0}$ положим $m_{-}=[m / 2], m_{+}=m-m_{-}$. Для $x \in \mathbb{Z}, n, z \in \mathbb{N}, x \geqslant 0$ определим также

$$
T_{z}^{n}(m, x)=\langle\underbrace{1, n, 1, n, \ldots, 1, n}_{m_{+} \text {пар чисел }}, n^{(x)}, z, \underbrace{n, 1, n, 1, \ldots, n, 1}_{m_{-} \text {пар чисел }}\rangle .
$$

Для натуральных $n$ и $S$ определим $U_{n}(S)$ как множество континуантов $\left\langle a_{1}, a_{2}, \ldots, a_{t}\right\rangle$ таких, что для всех $i$ таких, что $1 \leqslant i \leqslant t$, выполнено $a_{i} \leqslant n$, а также $\sum_{i=1}^{t} a_{t}=S$.

В статье [4] была доказана следующая теорема о минимальном значении континуанта на множестве $U_{n}(S)^{1}$.

ЛЕмма 2.1 [4; теорема 7]. При $n \geqslant 2, S \geqslant 2 n+2$ минималъный континуант на множестве $U_{n}(S)$ может быть найден по формуле

$$
\min \left(U_{n}(S)\right)=\min _{1 \leqslant z \leqslant n} T_{z}^{n}(m, x),
$$

где

$$
0 \leqslant x \leqslant n, \quad x \equiv 1-S(\bmod n+1), \quad m=\frac{S-n x-1}{n+1}, \quad m(z)>0,
$$

т.е. для нахождения минимума требуется найти оптимальное значение $z$.

Для $f$ натуральных чисел $h_{1}<h_{2}<\cdots<h_{f}$ и для их кратностей $p_{1}, p_{2}, \ldots, p_{f} \geqslant 1$ определим множество

$$
\left.W_{f}=W_{f}(\bar{h}, \bar{p})=\left\{\left(a_{1}, a_{2}, \ldots, a_{t}\right): a_{i} \in\left\{h_{1}, h_{2}, \ldots, h_{f}\right\}, \#\left\{i: a_{i}=h_{j}\right\}=p_{j}\right\}\right\},
$$

где $t$ равно $p_{1}+p_{2}+\cdots+p_{f}$.

Обозначим множество последовательностей вида $\left(a, b_{1}, a, b_{2}, a, \ldots, a, b_{n}, a\right)$ длины $2 n+1$, в которых на нечетных местах все элементы равны $a$, а последовательность $\left(b_{1}, b_{2}, \ldots, b_{n}\right)$, образуемая элементами, стоящими на четных местах, принадлежит множеству $W_{f}(\bar{h}, \bar{p})$ через $W_{f}^{a}(\bar{h}, \bar{p})$. Для $\bar{p}=\left(p_{1}, p_{2}, \ldots, p_{f}\right)$ целочисленные коэффициенты $p_{i}^{l}, p_{i}^{r}$ определяются по следующим правилам:

1) $p_{f}^{r}=\left[p_{f} / 2\right], p_{f}^{l}=p_{f}-p_{f}^{r}$;

2) при $i<f$ выполнено $p_{i}^{r}=p_{i}^{l}=p_{i} / 2$, если $p_{i}$ четно;

3 ) если $p_{i}$ нечетно и для всех $j>i$ верно, что $p_{j}$ четно, то $p_{i}^{r}=\left[p_{i} / 2\right], p_{f}^{l}=p_{i}-p_{i}^{r}$;

4) если $p_{i}$ нечетно и $j$ - минимальное число большее $i$ такое, что $p_{j}^{l} \neq p_{j}^{r}$, то $p_{i}^{l}$ и $p_{i}^{r}$ определяются из соотношения $\left(p_{j}^{l}-p_{j}^{r}\right)\left(p_{i}^{l}-p_{i}^{r}\right)=-1$.

Определим также параметры

$$
\lambda_{n}=\frac{n+\sqrt{n^{2}+4}}{2}, \quad \mu_{n}=\frac{n+2+\sqrt{n^{2}+4 n}}{2} .
$$

Положим $c_{0} \approx 0.203188$ - единственное решение уравнения

$$
x e^{2(1-x)}=1
$$

на отрезке $x \in[0,1 / 2]$.

\footnotetext{
${ }^{1} \mathrm{~B}$ статье было доказано более сильное утверждение, но нам будет достаточно сформулированной более простой и слабой формы.
} 


\section{3. Основные результаты.}

Tеорема 1. Пустъ $n \geqslant 2, S \geqslant(n+1)^{2} u S=(n+1) m+z_{0}, 0 \leqslant z_{0} \leqslant n, x$ находится из соотношения

$$
0 \leqslant x \leqslant n, \quad x \equiv 1-S(\bmod n+1) .
$$

Тогда минимальный континуант на множестве $U_{n}(S)$ имеет следующий вид:

- если $z_{0}>n / 2$, mо

$$
\min \left(U_{n}(S)\right)=T_{n}^{n}(m, x) ;
$$

- если $z_{0} \leqslant n / 2$, то минимум равен одному из двух континуантов:

$$
\min \left(U_{n}(S)\right)=T_{n}^{n}(m, x) \quad \text { или } \min \left(U_{n}(S)\right)=T_{z_{0}}^{n}(m, 0) .
$$

ЗАмечАниЕ. Существуют такие положительные константы $C_{1}, C_{2}$, что при $z_{0}>c_{0} n+C_{1}$ $\min \left(U_{n}(S)\right)$ равен $T_{n}^{n}(m, x)$, а при $z_{0}<c_{0} n-C_{2}$ он равен $T_{z_{0}}(m, 0)$. Значения $T_{n}^{n}(m, x)$ и $T_{z_{0}}^{n}(m, 0)$ можно вычислить асимптотически. Они равны

$$
T_{n}(m, x)=\frac{\mu_{n}^{m-n+z_{0}+2}}{\left(\mu_{n}+1\right)^{2}} \frac{\lambda_{n}^{n+3-z_{0}}}{\lambda_{n}^{2}+1}\left(1+O\left(\frac{1}{n}\right)\right) \quad \text { и } \quad T_{z_{0}}(m, 0)=\frac{\mu_{n}^{m+2}}{\left(\mu_{n}+1\right)^{2}}\left(z_{0}+O\left(\frac{1}{n}\right)\right) .
$$

Теорема 2. Минимальный континуант на множестве $W_{f}^{a}(\bar{h}, \bar{p})$ может быть найден по формуле

$$
\min \left(W_{f}^{a}(\bar{h}, \bar{p})\right)=\left\langle\left(a, h_{f}\right)^{\left(p_{f}^{l}\right)},\left(a, h_{f-1}\right)^{\left(p_{f-1}^{l}\right)}, \ldots,\left(a, h_{1}\right)^{\left(p_{1}\right)}, \ldots,\left(h_{f-1}, a\right)^{\left(p_{f-1}^{r}\right)},\left(h_{f}, a\right)^{\left(p_{f}^{r}\right)}\right\rangle .
$$

Теорема 3. Максимальный континуант на множестве $W_{f}^{a}(\bar{h}, \bar{p})$ при условии $p_{f-i}=$ $p_{i+1}$ для любого $i$ такого, что $0 \leqslant i \leqslant f-1$, может быть найден по формуле

$$
\begin{aligned}
\max & \left(W_{f}^{a}(\bar{h}, \bar{p})\right) \\
& =\left\langle\left(a, h_{1}, a, h_{f}\right)^{\left(p_{f}^{l}\right)},\left(a, h_{2}, a, h_{f-1}\right)^{\left(p_{f-1}^{l}\right)}, \ldots,\left(h_{f-1}, a, h_{2}, a\right)^{\left(p_{f-1}^{r}\right)},\left(h_{f}, a, h_{1}, a\right)^{\left(p_{f}^{r}\right)}\right\rangle .
\end{aligned}
$$

4. Набросок доказательства. Для доказательства теоремы 1 достаточно показать, что если произвольный континуант из множества $U_{n}(S)$ не имеет вид $T_{n}^{n}(m, x)$ или $T_{z_{0}}^{n}(m, 0)$, где $z_{0} \leqslant n / 2$, то для данного континуанта существует преобразование, переводящее его в меньший континуант. Лемма 2.1 показывает, что минимальный континуант лежит в множестве (5). Верна следующая техническая лемма.

Лемма 4.1. Для произвольных А и $C$ при $m \geqslant 0, n \geqslant 3 u 1 \leqslant z \leqslant n-1$ выполнены неравенства

$$
\begin{array}{lrr}
\left\langle A, 1, n, 1, n^{(m+1)}, z, n, 1, C\right\rangle\left\langle\left\langle A, 1, n^{(m+2)}, z+1, n, 1, C\right\rangle\right. & n p u & z \leqslant \frac{n}{2}, \\
\left.\left\langle A, 1, n, 1, n^{(m+1)}, z, n, 1, C\right\rangle\right\rangle\left\langle A, 1, n^{(m+2)}, z+1, n, 1, C\right\rangle & \text { npu } & z>\frac{n}{2} .
\end{array}
$$

Из (12) следует, что для любого континуанта $\langle M\rangle$ из (5) преобразование, переводящее его в меньший, существует при $n / 2<z<n$, а также при $1<z \leqslant n / 2$ и $x>0$. Это и означает, что минимум достигается только в континуантах вида $T_{n}^{n}(m, x)$ или $T_{z_{0}}^{n}(m, 0)$, где $z_{0} \leqslant n / 2$. Что и требовалось доказать. Утверждение о "разделяющей" константе $c_{0}$ доказывается из сравнения асимптотических формул (9).

Теоремы 2 и 3 доказываются аналогичными рассуждениями. Рассмотрим произвольный континуант из множества $W_{f}^{a}(\bar{h}, \bar{p})$ и докажем, что если он не равен (10) (в случае максимума - (11)), то для него существует преобразование, переводящее его в меньший 
(больший) континуант. В качестве таких преобразований мы будем использовать замены вида

$$
\langle A, \vec{B}, C\rangle \rightarrow\langle A, \overleftarrow{B}, C\rangle
$$

Такие преобразования удобны тем, что для них верна следующая лемма.

Лемма 4.2 [6]. Неравенство

$$
\langle A, \vec{B}, C\rangle>\langle A, \overleftarrow{B}, C\rangle
$$

выполнено тогда и толъко тогда, когда

$$
([0 ; \overleftarrow{A}]-[0 ; \vec{C}])([0 ; \vec{B}]-[0 ; \overleftarrow{B}])>0
$$

Применение данной леммы требует сравнивать цепные дроби, но в случае множества $W_{f}^{a}(\bar{h}, \bar{p})$ задача упрощается тем, что при рассмотрении замены

$$
\begin{aligned}
& \langle\underbrace{\left\langle, h_{i_{1}}, a, \ldots, a\right.}_{A}, \underbrace{h_{i_{k}}, a, \ldots, a, h_{i_{l}}}_{\vec{B}}, \underbrace{a, \ldots, a, h_{i_{n}}, a}_{C}\rangle \\
& \rightarrow \underbrace{\left\langle a, h_{i_{1}}, a, \ldots, a\right.}_{A}, \underbrace{h_{i_{k}}, a, \ldots, a, h_{i_{l}}}_{\overleftarrow{B}}, \underbrace{a, \ldots, a, h_{i_{n}}, a}_{C}\rangle
\end{aligned}
$$

при $h_{i_{k}} \neq h_{i_{l}}$ достаточно сравнить цепные дроби $[0 ; \overleftarrow{A}]$ и $[0 ; \vec{C}]$. Но в этих цепных дробях все неполные частные нечетного индекса равны $a$, поэтому из двух дробей $[0 ; \overleftarrow{A}]$ и $[0 ; \vec{C}]$ больше та, в которой первое отличающееся неполное частное больше. Если же одна из этих дробей является подходящей дробью к другой, то больше более короткая цепная дробь. Это приводит нас к следующему принципу, применение которого позволяет доказать теоремы 2 и 3. Его можно неформально сформулировать следующим образом.

Для увеличения континуанта из множества $W_{f}^{a}(\bar{h}, \bar{p})$ необходимо добиватвся, чтобы большие неполные частные стояли рядом с менъшими неполными частными. Для уменьшения континуанта из данного множества необходимо, напротив, ставить большие неполные частные рядом с большими, а менъшие рядом с менъшими.

\section{СПИСОК ЦИТИРОВАННОЙ ЛИТЕРАТУРЫ}

[1] А. А. Душистова, Н. Г. Мощевитин, Фундамент. и прикл. матем., 16:6 (2010), 33-44. [2] A. A. Dushistova, I. D. Kan, N. G. Moshchevitin, J. Math. Anal. Appl., 401:2 (2013), 774-794. [3] Д. Р. Гайфулин, Алгебра и анализ, 27:1 (2015), 74-124. [4] И. Д. Кан, Фундамент. и прикл. матем., 16:6 (2010), 95-108. [5] Р. Грэхем, Д. Кнут, О. Паташник, Конкретная математика. Основание информатики, Мир, М., 1998. [6] И. Д. Кан, Дискрет. матем., 12:3 (2000), 72-75.

\section{Д. Р. Гайфулин}

